\title{
Adult Neurogenesis Is Associated with the Maintenance of a Stereotyped, Learned Motor Behavior
}

\author{
Carolyn L. Pytte, ${ }^{1}$ Shanu George, ${ }^{1}$ Shoshana Korman, ${ }^{1}$ Eva David, ${ }^{1}$ Diane Bogdan, ${ }^{1}$ and John R. Kirn ${ }^{2}$ \\ ${ }^{1}$ Department of Psychology, Queens College, City University of New York, Flushing, New York 11367, and ${ }^{2}$ Department of Biology, Wesleyan University, \\ Middletown, Connecticut 06459
}

Adult neurogenesis is thought to provide neural plasticity used in forming and storing new memories. Here we show a novel relationship between numbers of new neurons and the stability of a previously learned motor pattern. In the adult zebra finch, new projection neurons are added to the nucleus HVC and become part of the motor pathway for producing learned song. However, new song learning occurs only in juveniles and the behavioral impact of adding new neurons to HVC throughout life is unclear. We report that song changes after deafening are inversely correlated with the number of new neurons added to HVC, suggesting that adult neurogenesis in this context may contribute to behavioral stability. More broadly, we propose that new neuron function may depend on the site of integration and can vary as widely as promoting, or restricting, behavioral plasticity.

\section{Introduction}

Our current understanding of the function of adult neurogenesis is that new neurons provide a plastic substrate for forming and storing new memories (Deng et al., 2010; Aimone et al., 2011; Lazarini and Lledo, 2011; Ming and Song, 2011). This model is primarily based on studies of the mammalian hippocampus and olfactory bulb, as well as the avian vocal control system in birds that continue to learn new songs in adulthood. Yet, in the zebra finch, neuron addition continues in the song control system throughout life (Alvarez-Buylla et al., 1990), long after song learning is complete. Here we propose a novel role for new neurons in the avian song motor pathway, in which newly incorporated neurons are associated with maintaining the complex learned song motor pattern after complete auditory deprivation. This raises the possibility that new neurons may restrict behavioral plasticity, and the function of new neurons may depend on the brain region into which they are incorporated.

New projection neurons are added throughout life into the telencephalic nucleus HVC (proper name) of the songbird and synapse in the premotor robust nucleus of the acropallium (RA), becoming part of the vocal motor pathway (VMP) for song production (Alvarez-Buylla et al., 1990; Hahnloser et al., 2002). The stereotyped song structure of adult zebra finches gradually deteriorates after deafening (Nordeen and Nordeen, 1992) and the rate of degradation varies greatly among individuals (Fig. $1 A$ ).

Received 0ct. 25, 2011; revised March 23, 2012; accepted March 29, 2012.

Author contributions: C.L.P. designed research; C.L.P., S.G., S.K., and E.D. performed research; C.L.P., S.G., S.K., and D.B. analyzed data; C.L.P. and J.R.K. wrote the paper.

This work was supported by a SOMAS-URM grant from the Howard Hughes Medical Institute (Grant 52006292 to Davidson College), a National Science Foundation grant (DUE-0426266 to Dr. Julio J. Ramirez), PSCREG-39-1018 and NIH R03 NS063182 (to C.L.P.).

The authors declare no financial conflict of interest.

Correspondence should be addressed to Carolyn L. Pytte, Psychology Department, Queens College, CUNY, 65-30 Kissena Boulevard, Flushing, NY 11367. E-mail: Carolyn.pytte@qc.cuny.edu.

DOI:10.1523/JNEUROSCI.5385-11.2012

Copyright $\odot 2012$ the authors $\quad 0270-6474 / 12 / 327052-06 \$ 15.00 / 0$
The reasons for song deterioration, and for individual differences, are poorly understood. One idea is that deafening-induced song changes result from new neuron incorporation into HVC in the absence of singing-related auditory feedback, which may normally guide appropriate firing and connectivity of incoming neurons (Scott et al., 2000, 2007). However, the VMP is not sufficient to bring about vocal changes after deafening and change requires input from the anterior forebrain pathway (AFP), which converges on the VMP (Brainard and Doupe, 2000). This suggests that new VMP neurons incorporated in the absence of auditory feedback may not disrupt existing motor circuits as previously proposed. Instead, if new neurons serve to enhance VMP function, they may contribute to song stability. Here we explored this possibility by comparing neuron addition in $\mathrm{HVC}$ and song maintenance post-deafening.

\section{Materials and Methods}

Animals

Methods were approved by the Queens College Institutional Animal Care and Use Committee. Subjects were adult male zebra finches 4-7 months old at the time of cell birth-dating. Birds were hatched in the Queens College breeding colony and kept with their parents until 70-90 $\mathrm{d}$ of age. Thereafter, birds were housed in a single large cage $(n=9)$ within auditory and visual contact of birds of both sexes until 1 month before cell birth-dating. Birds were then housed individually and maintained on a 13:11 h light:dark cycle.

\section{Cell birth-dating}

Mitotically active cells were labeled using bromodeoxyuridine (BrdU; three times a day for $3 \mathrm{~d} ; 74 \mu \mathrm{l}$ of $10 \mathrm{mg} / \mathrm{ml}$ in tris-buffered saline, $\sim 0.074$ $\mathrm{mg} / \mathrm{g})$.

\section{Surgery}

Two days after the last BrdU injection, birds were deafened by bilateral surgical removal of the cochlea, as in Pytte et al. (2010).

\section{Histology}

Twenty-eight days after the last BrdU injection, birds were overdosed with sodium pentobarbital (Euthasol; Henry Shein) and perfused with 
A

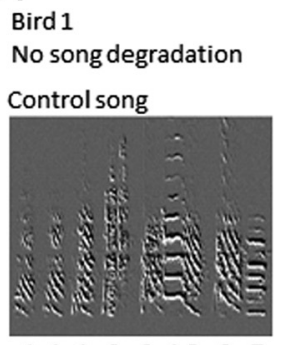

$\begin{array}{llllllll}1 & 1 & 1 & 2 & 3 & 45 & 6 & 7\end{array}$
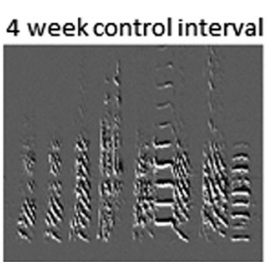

$\begin{array}{lllllllll}1 & 1 & 1 & 2 & 3 & 4 & 5 & 6 & 7\end{array}$ Accuracy score $99 \%$

B
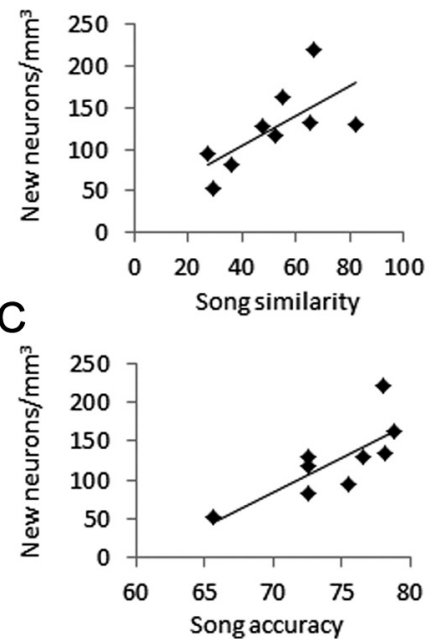

Bird 3

High song degradation

Pre-deaf song

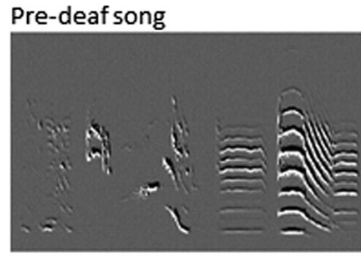

$\begin{array}{lllllllll}1 & 2 & 3 & 4 & 5 & 6 & 7 & 89 & 10\end{array}$

\section{4 weeks after deafening}

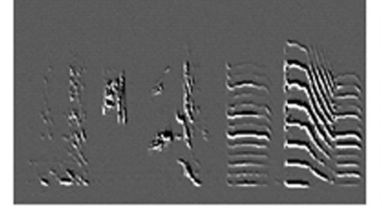

$\begin{array}{llllllll}12 & 23 & 4 & 5 & 6 & 7 & 89 & 10\end{array}$

Accuracy score $96 \%$

D

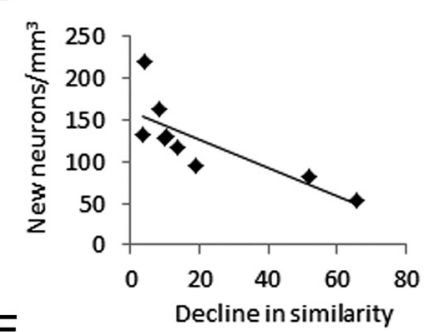

$\mathrm{E}$

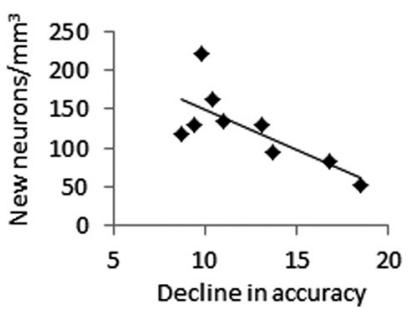

Figure 1. A, Spectral derivatives of the songs of three birds, generated with Sound Analysis Pro. The song of hearing control Bird 1 does not change in song structure 4 weeks after the initial recording (left). The song of Bird 2 shows a low degree of song degradation 4 weeks after deafening (middle). The song of Bird 3 demonstrates a high degree of post-deafening song degradation at 4 weeks (right). Song notes are numbered below the figures to show same notes across recordings. Accuracy scores are a measure of the degree to which post-4 week songs are acoustically the same as the pre-deaf songs. $\boldsymbol{B}-\boldsymbol{E}$, New neuron number was positively correlated with song stability measured as similarity $(\boldsymbol{B})$, and accuracy $(\boldsymbol{C})$ between pre-deaf and post-deaf songs. Post-deaf declines in similarity $(\boldsymbol{D})$ and accuracy $(\boldsymbol{E})$ were inversely correlated with new neuron numbers.

0.1 м PBS followed by $4 \%$ paraformaldehyde. The brains were processed for immunocytochemistry and double-labeled with antibodies to BrdU and the neuron-specific protein $\mathrm{Hu}$, as described previously (Pytte et al., 2010, 2011).

\section{Microscopy}

HVC area measurements and cell counts were performed using a fluorescence microscope and mapping software (Olympus BX51; Lucivid microprojection, Neurolucida, MicroBrightField). HVC was traced in 10 evenly distributed sections per bird. BrdU-labeled cells were seen with a fluorescein isothiocyanate (FITC) filter and Hu-labeled cells were visualized with a rhodamine filter. Double-labeled cells were identified using a dual FITC-rhodamine filter. Traced HVC areas were multiplied by section thickness and sampling interval to estimate HVC volumes. New neuron densities per cubic millimeter were calculated by dividing the numbers of labeled cells by the volumes sampled. Estimates of total new neuron numbers in HVC were calculated by multiplying the densities of $\mathrm{BrdU}+/ \mathrm{Hu}+$ cells by the total estimated HVC volumes. No corrections were made for cell splitting because we were interested in relative rather than absolute differences in neuron numbers (Guillery and Herrup, 1997). We found the same results using measures of new neuron density and estimated total number of new neurons within HVC. We report only new neuron density.

Song recording and analysis

Birds were housed individually in soundattenuated chambers and recorded continuously with sound-activated Avisoft Recorder (Avisoft Bioacoustics). Song stereotypy before deafening and changes in structure after deafening were computed using Sound Analysis Pro (Tchernichovski et al., 2000), which quantifies and compares the spectral structure of sound pairs based on the acoustic features of pitch, frequency modulation, amplitude modulation, Wiener entropy, and goodness of pitch. Two measures of song stability were generated: percentage similarity and accuracy.

Percentage similarity (termed here "similarity") is the percentage of the deaf song that meets a similarity threshold compared with the pre-deaf song. The accuracy score quantifies the magnitude of song likeness between two songs. We used both scores to determine whether measures of song stability calculated by different algorithms were independently correlated with the new neuron numbers. For all song datasets, we selected the first 100 highquality songs produced after the first hour of the day, visualized with Raven software (Cornell Lab of Ornithology).

Comparison between pre-deaf song and postdeaf song. We quantified the similarity between pre-deaf and post-deaf song structure by comparing 100 pre-deaf and 100 post-deaf songs, generating pre-to-post similarity and accuracy scores using a symmetrical matrix analysis with Sound Analysis Pro, eliminating all redundant comparison pairs. We used the symmetrical analysis because it is a more sensitive measure of song likeness than the alternative asymmetrical analysis when syllables are added or deleted in the deaf song from the pre-deaf song.

Normalized change in song structure. We also measured the changes in song acoustic structure relative to pre-deaf baseline similarity and accuracy scores. To do this, we calculated similarity and accuracy scores between 100 songs recorded on each of $2 \mathrm{~d}$ pre-deafening. Songs were compared across $2 \mathrm{~d}$ to parallel the pre-to-post song comparisons. Individual pre-deaf similarity and accuracy scores ranged from 60 to 95 (mean, 72) and 83 to 97 (mean, 88), respectively. We subtracted individual pre-to-post-deaf values from these baseline values, providing a measure of song degradation normalized to natural pre-deaf song stereotypy. The degree of pre-deaf song stereotypy did not predict the extent of song degradation after deafening (similarity score: $R^{2}=0.033, F=0.238, p=$ 0.640; accuracy score: $R^{2}=0.208, F=1.836, p=0.218$ ).

Percentage change in song structure. The percentage of change in song stereotypy was calculated by dividing the values of normalized song change (described above) by the pre-deaf values of similarity and accuracy scores.

Change in acoustic features. Sound Analysis Pro also measures amplitude, pitch, frequency modulation, amplitude modulation, Weiner entropy, pitch goodness, mean frequency, and the variances of these features. For each bird, we computed the mean values for these measures in one of the pre-deaf sets of 100 songs used above and also in the 100 post-deaf songs and assessed the changes in feature values. Of 
these features, only three changed significantly after deafening: Weiner entropy, entropy variance, and pitch.

Singing rate. Songs were counted using the "Scan for Template Spectrogram Patterns" batch processing function in Avisoft SASLab Pro, which tallies occurrences of user-defined acoustic templates in a large database of recordings. We generated templates using syllable sequences unique to each bird's song.

We found a significant correlation between individual singing rates across 2 nonsuccessive days before deafening, suggesting that singing rate is a relatively stable trait in individually housed, unmanipulated birds (Spearman's rank correlation, $r \mathrm{~s}=0.867, p<0.05$ ). We computed mean pre-deaf singing rates per daylight hour based on recordings over $2 \mathrm{~d}$.

However, singing rate was not consistent across individuals after deafening. High singers and low singers maintained their relative singing rates throughout the experiment, but intermediately ranked singers switched ranks after deafening and throughout the post-deaf period. This prompted us to quantify all songs produced after deafening and compare these values with song maintenance and the new neuron number.

Given that singing rates after deafening were highly variable, we also asked whether singing rates corresponding to different life stages of our labeled neuron population were correlated with song maintenance and numbers of new HVC neurons. New neurons begin to arrive in HVC by day 7 after mitosis and peak in numbers at approximately day 14 (J.R. Kirn, unpublished observations). At day 14, neuron survival is increased by exposure to the growth factor BDNF (Alvarez-Borda et al., 2004), which is upregulated in canaries during singing ( $\mathrm{Li}$ et al., 2000). In canaries, HVC neuron number is decreased in birds with suppressed song production between days 25 and 33 after cell birth-dating (Li et al., 2000). Based on the timing of these particular neuronal events, we compared new neuron counts and song degradation with singing rates at post-deaf days $7,14,27$, and throughout days 14-17.

Effects of deafening on new neuron survival

To determine whether deafening itself impacts new neuron survival under our experimental conditions, we compared the density and estimated total new neuron numbers in HVC between another set of age-matched, individually housed deafened birds and hearing controls $(n=8$ for each group, aged 3-5 months). All methods and housing were as described above. Control birds received a sham surgery without opening the bony cochlea casing. We found the same results using either measure of new neurons and report only new neuron densities.

\section{Results}

Pre-to-post measures of song similarity and accuracy were positively correlated with the density of new neurons in HVC (similarity score: $R^{2}=0.461, F=5.997, p=0.044$; accuracy score: $R^{2}=0.583, F=9.779, p=0.017$; Fig. $\left.1 B, C\right)$. Normalized changes in song structure after deafening were inversely correlated with new neuron numbers (similarity score: $R^{2}=0.614$, $F=11.129, p=0.012$; accuracy score: $R^{2}=0.541, F=8.243, p=$ 0.024 ; Fig. $1 D, E)$. Higher numbers of new HVC neurons corresponded to less song change after deafening. Normalized changes in song were independent from pre-to-post similarity and accuracy scores $\left(R^{2}=0.250, F=2.333, p=0.170 ; R^{2}=0.176\right.$, $F=1.50, p=0.170$, respectively). Percentage changes in song acoustic structure after deafening were similarly inversely correlated with new neuron addition (percentage change in similarity score: $R^{2}=0.695, F=15.966, p=0.005$; percentage change in accuracy: $R^{2}=0.556, F=8.772, p=0.021$; data not shown). There was no correlation between new neuron numbers and total HVC volume $(p>0.05)$.

Of the acoustic features measured, Weiner entropy, entropy variance, and pitch changed after deafening. For each, the magnitude of change was inversely correlated with the numbers of new neurons in HVC. Across all birds, entropy values increased significantly after deafening (paired $t$ test, $t=4.163$, two-tailed $p=0.005$ ) and birds with more new neurons showed less of an
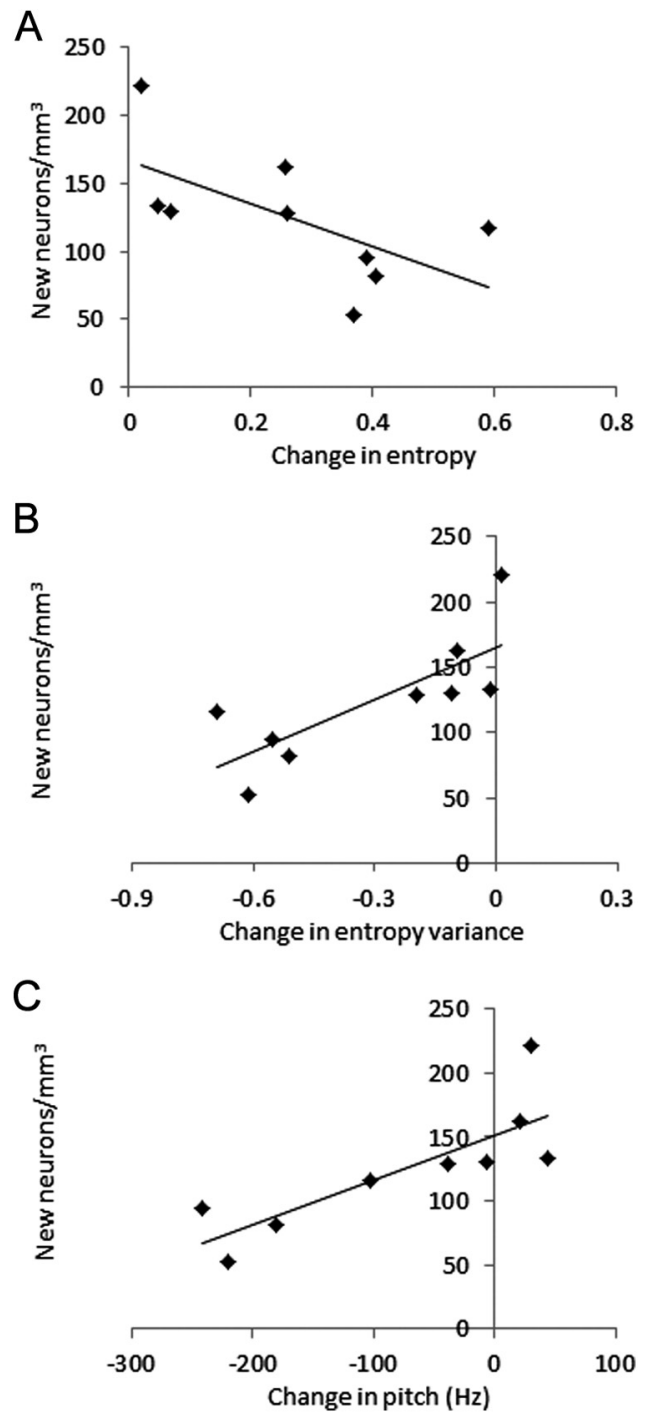

Figure 2. $\boldsymbol{A}-\boldsymbol{C}$, Post-deafening day 27 changes in the song features Weiner entropy $(\boldsymbol{A})$, entropy variance $(\boldsymbol{B})$, and pitch $(\boldsymbol{C})$ were negatively correlated with new neuron numbers. The zero point in all graphs represents no change between pre- and post-deaf song feature.

increase in entropy post-deafening $\left(R^{2}=0.515, F=7.429, p=\right.$ 0.029; Fig. $2 A$ ). Wiener entropy corresponds inversely to tonal or harmonic structure. Acoustic noisiness, or decreased structure, regularly increases in the songs of deaf birds. Entropy variance decreased significantly after deafening (paired $t$ test, $t=3.862$; two-tailed, $\mathrm{p}=0.005)$, and birds with more new neurons showed less of a decrease in entropy variance $\left(R^{2}=0.688, F=15.420\right.$, $p=0.006$; Fig. $2 B$ ). Entropy variance increases with increased temporal structure and also increases developmentally as notes become increasingly structured and stereotyped (Derégnaucourt et al., 2005). Coupled with increased mean Weiner entropy, decreased entropy variance reflects a decrease in tonality. After deafening, pitch decreased and birds with more new neurons had less of a pitch decrease $\left(R^{2}=0.660, F=13.569 p=0.008\right.$; Fig. $2 C)$. There was no correlation between bird age and new neuron number or any of our measures of song change.

Singing is the result of activity of $\mathrm{HVC}_{\mathrm{RA}}$ projection neurons (Hahnloser et al., 2002; Scotto-Lomassese et al., 2007) and may promote new neuron survival (Li et al., 2000). Therefore, we assessed whether song degradation and neuron number were correlated with singing rate. We found an inverse trend between 

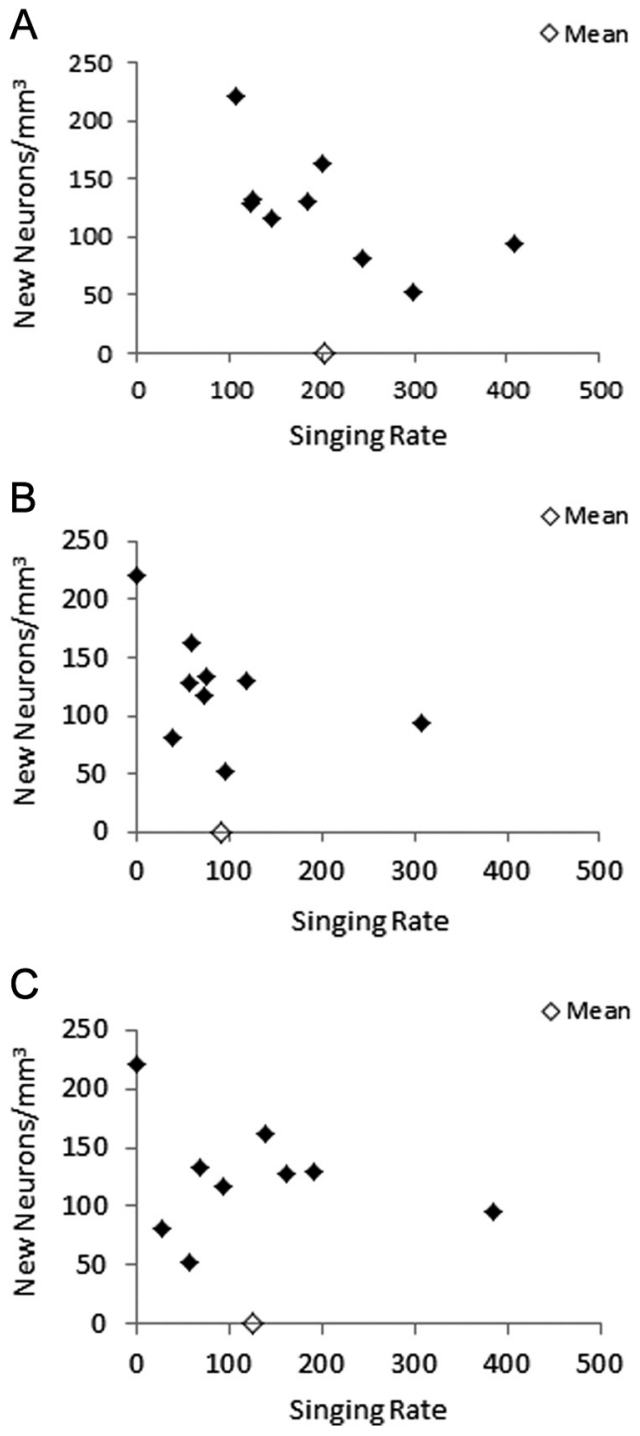

Figure 3. $\boldsymbol{A}$, There was an inverse trend between new neurons and pre-deaf singing. We found no correlations between new neurons and singing rates after deafening at any time point sampled, or throughout the entire post-deaf period. $\boldsymbol{B}, \boldsymbol{C}$, Shown are new neurons corresponding to singing rates on post-deaf days $14(\boldsymbol{B})$ and $27(\boldsymbol{C})$.

new neuron numbers and quantity of singing before deafening $\left(R^{2}=0.413, F=4.923, p=0.062\right.$; Fig. $\left.3 A\right)$ and no correlations between new neuron numbers and singing rates after deafening or changes in singing rates after deafening ( $p>0.05$ for all). We then compared new neuron numbers and singing rates at specific times (post-deaf days 7, 14, 27, and 14-17) that correspond to time points at which young neurons are likely to be influenced by song production and/or song feedback. We found no relations within these datasets ( $p>0.05$ for all; Fig. $3 B, C)$.

Finally, we found no associations between singing rates before or after deafening and measures of post-deaf song similarity, accuracy, or post-deaf changes in acoustic features $(p>0.05$ for all). Thus, our correlations between numbers of new neurons and song stability do not seem to be mediated by amount of singing. However, HVC neural activity does not always result in audible song, as it can occur, for example, during sleep (Crandall et al., 2007) and therefore singing rate may be an imprecise measure of activity in HVC.

We also assessed new neuron addition to HVC in a separate set of individually housed age-matched deaf and control birds. We

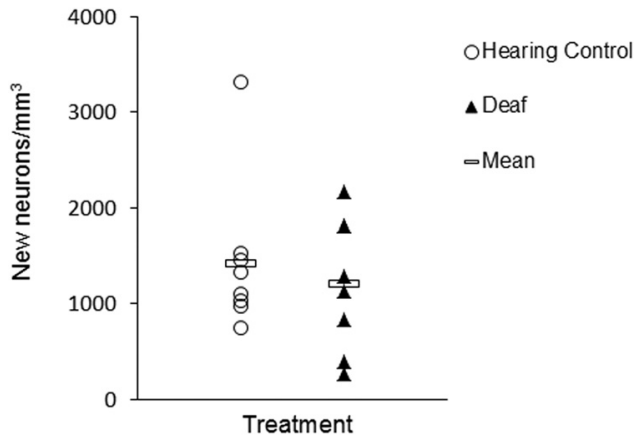

Figure 4. There was no difference in new neuron number between age-matched deafened birds and hearing controls.

found no difference in new HVC neurons between these deaf and hearing birds ( $t$ test, $t=0.192$; two-tailed, $p=0.851$; Fig. 4 ), suggesting that factors related to auditory deprivation or the act of deafening, such as stress or changes in behavior, do not alter new neuron number.

\section{Discussion}

Our finding that song maintenance after deafening was positively correlated with the number of new neurons incorporated into the song motor pathway in the adult brain is the first instance in which new neuron numbers have been associated with decreased behavioral plasticity. It is possible that new neurons are not functionally active in chronically deaf birds; however, the association nevertheless raises the intriguing suggestion that new neurons in this context may function in stabilizing an existing motor behavior. This is consistent with the finding that the impact on song of botox injections into the syrinx was smallest, and song recovery was greatest, in birds with the highest rates of HVC neuron addition (Pytte et al., 2011).

Prior work suggested that deafening had a dramatic effect on neuron addition in the adult zebra finch (Wang et al., 1999). However, in subsequent studies, we found that deafening had minor effects on neuron addition in HVC (Hurley et al., 2008) which were only apparent when deaf and hearing birds were allowed to interact in a group cage. We believe that social factors other than singing played an important role in this context. New neuron number in group-housed deaf birds did not differ from that of separately caged group-housed hearing birds (in preparation). Likewise, individually housed deaf and hearing birds in the present study did not differ in new neuron addition.

Stress and exercise influence new neuron survival in the hippocampus (Snyder et al., 2009) and individual differences in these factors may have contributed to differences in new neuron number and perhaps also to differences in song stability. Individual differences in circulating testosterone $(\mathrm{T})$ might also independently influence song maintenance and neuron addition (Williams et al., 2003). However, T levels are generally positively correlated with singing rates in canaries (Li et al., 2000; AlvarezBorda and Nottebohm, 2002), and we found either a negative trend (between pre-deaf singing rate and new neuron number) or no correlations (post-deaf) between singing rates and song maintenance or new neuron number, consistent with Pytte et al. (2011). Although perhaps a correlation between singing rates and new neurons requires auditory feedback. Regardless, androgens cannot be ruled out as a contributing factor to our results, particularly since we do not know how T levels relate to singing rate in zebra finches or in deafened birds. A more direct appraisal of 
the potential relationship between $\mathrm{T}$ and other hormones including glucocorticoids, neuron addition, and song degradation is warranted.

We did not find a correlation between bird age and song degradation or new neurons, which is consistent with other reports that found an age-related decrease in post-deaf song degradation and decline in new neurons only when a larger age range was considered (Lombardino and Nottebohm, 2000; Brainard and Doupe, 2001; Wang et al., 2002). However, Pytte et al. (2007) showed that in aging birds, neuron addition declined as song becomes increasingly stereotyped. This relationship seems at odds with the positive association shown here between increased song stability and increased neuron addition, although it is not clear whether normal song stereotypy (Pytte et al., 2007) and post-deafening song stability are mechanistically related. More directly related to our study, decreased neuron addition with age (Wang et al., 2002; Walton et al., 2012), coupled with an agerelated decline in post-deafening song degradation (Lombardino and Nottebohm, 2000; Brainard and Doupe, 2001), suggests that the high numbers of new neurons seen in younger adults correspond to post-deaf song instability, rather than stability, as our current results indicate. An explanation for the apparent contradiction between the present results and previous studies across a large age range may lie in the relationship between neuron addition and the total number of $\mathrm{HVC}_{\mathrm{RA}}$ neurons.

$\mathrm{HVC}$ continues to accumulate new $\mathrm{HVC}_{\mathrm{RA}}$ neurons throughout life (Walton et al., 2012) and it may be the case that total $\mathrm{HVC}_{\mathrm{RA}}$ neuron number is the variable most important for agerelated increases in song stability after deafening. With this model, rates of neuron addition may be positively correlated with the total $\mathrm{HVC}_{\mathrm{RA}}$ neuron number, and the larger the latter population, the greater the stability of song after deafening. Examination of previous work (Lombardino and Nottebohm, 2000; Wang et al., 2002) indicates that when comparing young (4-7 months) and older ( $>12$ months) adults, within-age variability in deafening effects and rates of new neuron addition is smaller in magnitude than between-age changes in these attributes. Thus, it is possible that even in old birds there is a positive correlation between rates of neuron addition and song preservation, even though overall rates of neuron addition and song degradation are much lower than in young birds. If true, song stability at any age would be greatest for birds with highest rates of neuron addition and total $\mathrm{HVC}_{\mathrm{RA}}$ neuron number.

Although speculative, this model is supported by all correlations among new neurons, age, and post-deafening song degradation known to date, including the present findings. Our findings are also consistent with the suggestion of Thompson and Johnson (2007) that the relative contributions of the VMP and the AFP promote song stereotypy and variability, respectively. Thompson et al. (2007) proposed that deafening results in increased relative influence of AFP, consistent with Brainard and Doupe (2000). Direct support of the role of AFP in postdeafening song degradation was provided by lesioning the output of the AFP, which blocked (Brainard and Doupe, 2000), or reversed some degree of post-deafening song degradation (Nordeen and Nordeen, 2010). Moreover, deafening altered dendritic spines and synaptic strength of the HVC neuron population that project to the AFP, and not $\mathrm{HVC}_{\mathrm{RA}}$ neurons (Tschida and Mooney, 2012). However, blocking AFP output to the VMP in deaf birds does not fully restore severely degraded song to its pre-deaf structure (Nordeen and Nordeen, 2010), suggesting that deafening does also alter the VMP, perhaps within RA, and perhaps reflecting VMP consolidation of AFP output, as described by Andalman and Fee (2009) and Warren et al. (2011). Our study further suggests that naturally occurring high numbers of new (or total) $\mathrm{HVC}_{\mathrm{RA}}$ neurons may contribute to the stabilizing influence of the VMP on song production.

Young neurons specifically may preserve post-deaf song structure better than old $\mathrm{HVC}_{\mathrm{RA}}$ neurons, perhaps by increased firing accuracy required for maintaining song timing (Long and Fee, 2008) or by providing plasticity necessary to continually fine tune drift in the song premotor pattern. Regardless of whether new neurons are better than, or equivalent to, old neurons in maintaining song stability, the current results are counterintuitive in light of our understanding of hippocampal neurogenesis and new neurons in birds who continue to learn new songs, and raise new ideas about the functions of adult neurogenesis across brain regions. The finding that new neurons can impart stability when incorporated into the VMP of the zebra finch suggests that neurogenesis may not universally play a role in plasticity and learning, but that function may depend on the circuit receiving new neurons-in this case, a circuit that is necessary for song stability.

\section{References}

Aimone JB, Deng W, Gage FH (2011) Resolving new memories: a critical look at the dentate gyrus, adult neurogenesis, and pattern separation. Neuron 70:589-596.

Alvarez-Borda B, Nottebohm F (2002) Gonads and singing play separate, additive roles in neuron recruitment in adult canary brain. J Neurosci 22:8684-8690.

Alvarez-Borda B, Haripal B, Nottebohm F (2004) Timing of brain-derived neurotrophic factor exposure affects life expectancy of new neurons. Proc Natl Acad Sci U S A 101:3957-3961.

Alvarez-Buylla A, Kirn JR, Nottebohm F (1990) Birth of projection neurons in adult avian brain may be related to perceptual or motor learning. Science 249:1444-1446.

Andalman AS, Fee MS (2009) A basal ganglia-forebrain circuit in the songbird biases motor output to avoid vocal errors. Proc Natl Acad Sci U S A 106:12518-12523.

Brainard MS, Doupe AJ (2000) Interruption of a basal ganglia-forebrain circuit prevents plasticity of learned vocalizations. Nature 404:762-766.

Brainard MS, Doupe AJ (2001) Postlearning consolidation of birdsong: stabilizing effects of age and anterior forebrain lesions. J Neurosci 21:2501-2517.

Crandall SR, Adam M, Kinnischtzke AK, Nick TA (2007) HVC neural sleep activity increases with development and parallels nightly changes in song behavior. J Neurophysiol 98:232-240.

Deng W, Aimone JB, Gage FH (2010) New neurons and new memories: how does adult hippocampal neurogenesis affect learning and memory? Nat Rev Neurosci 11:339-350.

Derégnaucourt S, Mitra PP, Fehér O, Pytte C, Tchernichovski O (2005) How sleep affects the developmental learning of bird song. Nature 433:710-716.

Guillery RW, Herrup K (1997) Quantification without pontification choosing a method for counting objects in sectioned tissues. J Comp Neurol 386:2-7.

Hahnloser RH, Kozhevnikov AA, Fee MS (2002) An ultrasparse code underlies the generation of neural sequences in a songbird. Nature 419: 65-70.

Hurley P, Pytte C, Kirn JR (2008) Nest of origin predicts adult neuron addition rates in the vocal control system of the zebra finch. Brain Behav Evol 71:263-270.

Lazarini F, Lledo PM (2011) Is adult neurogenesis essential for olfaction? Trends Neurosci 34:20-30.

Li XC, Jarvis ED, Alvarez-Borda B, Lim DA, Nottebohm F (2000) A relationship between behavior, neurotrophin expression, and new neuron survival. Proc Natl Acad Sci U S A 97:8584-8589.

Lombardino AJ, Nottebohm F (2000) Age at deafening affects the stability of learned song in adult male zebra finches. J Neurosci 20:5054-5064.

Long MA, Fee MS (2008) Using temperature to analyse temporal dynamics in the songbird motor pathway. Nature 456:189-194. 
Ming GL, Song H (2011) Adult neurogenesis in the mammalian brain: significant answers and significant questions. Neuron 70:687-702.

Nordeen KW, Nordeen EJ (1992) Auditory feedback is necessary for the maintenance of stereotyped song in adult zebra finches. Behav Neural Biol 57:58-66.

Nordeen KW, Nordeen EJ (2010) Deafening-induced vocal deterioration in adult songbirds is reversed by disrupting a basal ganglia-forebrain circuit. J Neurosci 30:7392-7400.

Pytte CL, Gerson M, Miller J, Kirn JR (2007) Increasing stereotypy in adult zebra finch song correlates with a declining rate of adult neurogenesis. Dev Neurobiol 67:1699-1720.

Pytte CL, Parent C, Wildstein S, Varghese C, Oberlander S (2010) Deafening decreases neuronal incorporation in the avian auditory region of the caudomedial nidopallium (NCM). Behav Brain Res 211:141-147.

Pytte C, Yu YL, Wildstein S, George S, Kirn JR (2011) Adult neuron addition to the zebra finch song motor pathway correlates with the rate and extent of recovery from botox-induced paralysis of the vocal muscles. J Neurosci 31:16958-16968.

Scott LL, Nordeen EJ, Nordeen KW (2000) The relationship between rates of HVc neuron addition and vocal plasticity in songbirds. J Neurobiol 43:79-88.

Scott LL, Nordeen EJ, Nordeen KW (2007) LMAN lesions prevent song degradation after deafening without reducing HVC neuron addition. Dev Neurobiol 67:1407-1418.

Scotto-Lomassese S, Rochefort C, Nshdejan A, Scharff C (2007) HVC interneurons are not renewed in adult male zebra finches. Eur J Neurosci 25:1663-1668.

Snyder JS, Glover LR, Sanzone KM, Kamhi JF, Cameron HA (2009) The effects of exercise and stress on the survival and maturation of adultgenerated granule cells. Hippocampus 19:898-906.

Tchernichovski O, Nottebohm F, Ho CE, Pesaran B, Mitra PP (2000) A procedure for an automated measurement of song accuracy. Anim Behav 59:1167-1176.

Thompson JA, Johnson F (2007) HVC microlesions do not destabilize the vocal patterns of adult male zebra finches with prior ablation of LMAN. Dev Neurobiol 67:205-218.

Thompson JA, Wu W, Bertram R, Johnson F (2007) Auditorydependent vocal recovery in adult male zebra finches is facilitated by lesion of a forebrain pathway that includes the basal ganglia. J Neurosci $27: 12308-12320$.

Tschida KA, Mooney R (2012) Deafening drives cell-type-specific changes to dendritic spines in a sensorimotor nucleus important to learned vocalizations. Neuron 73:1028-1039.

Walton C, Pariser E, Nottebohm F (2012) The zebra finch paradox: song is little changed, but number of neurons doubles. J Neurosci 32:761-774.

Wang N, Aviram R, Kirn JR (1999) Deafening alters neuron turnover within the telencephalic motor pathway for song control in adult zebra finches. J Neurosci 19:10554-10561.

Wang N, Hurley P, Pytte C, Kirn JR (2002) Vocal control neuron incorporation decreases with age in the adult zebra finch. J Neurosci 22 : $10864-10870$.

Warren TL, Turner EC, Charlesworth JD, Brainard MS (2011) Mechanisms and time course of vocal learning and consolidation in the adult songbird. J Neurophys 106:1806-1821.

Williams H, Connor DM, Hill JW (2003) Testosterone decreases the potential for song plasticity in adult male zebra finches. Horm Behav 44:402412 . 